\title{
UTILIZAÇÃO DE AGREGADOS RECICLADOS PARA A PRODUÇÃO DE CONCRETOS: UMA REVISÃO BIBLIOMÉTRICA
}

Utilization of recycled aggregates to produce concrete: a bibliometric review

Paulo Roberto Dutra'; Michel Ostroski²; Gilson Francisco Paz Soares ${ }^{3}$

${ }^{1}$ Universidade Regional Integrada do Alto Uruguai e das Missões - URI Erechim, Erechim, RS, Brasil.

E-mail: paulo_rdutra@outlook.com; michelostr@gmail.com; gilsonfps@uricer.edu.br

Data do recebimento: 27/01/2021 - Data do aceite: 28/05/2021

RESUMO: Apesar dos avanços, um dos grandes problemas enfrentados pelo setor da construção civil ainda é o gerenciamento de resíduos. Por outro lado, algumas pesquisas mostram a viabilidade de utilização de agregados reciclados para a produção de concreto. Assim, o presente trabalho busca investigar o cenário das produções científicas, em todo o mundo, sobre a incorporação de resíduos no concreto. Para isso, realizou-se uma revisão bibliométrica a partir das bases de dados Scopus - Web of Science e de termos de busca adequada à ideia inicial. A análise abrangeu os seguintes parâmetros: produção anual acumulada, relevância e produtividade de periódicos e autores, e documentos mais citados globalmente. Após a sumarização dos dados, pôde-se comprovar que o uso de agregados reciclados em concretos é uma tendência crescente, com aproximadamente metade da produção concentrada nos dois últimos anos. Destaca-se, também, a dispersão das publicações entre vários autores e periódicos, de modo que há um pequeno núcleo que concentra as ações sobre o tema. Assim, devem ser estes periódicos alvo de investigações para futuras publicações.

Palavras-chave: Agregados reciclados. Concreto. Revisão bibliométrica.

ABSTRACT: Despite the advances, one of the civil construction sector's significant problems is still the waste management. On the other hand, research shows that it is possible to use recycled aggregates to produce concrete. Thus, the present work investigates the scenario of scientific productions worldwide about incorporating waste into the concrete. For this, a bibliometric review 
was carried out from the Scopus - Web of Science databases and searching appropriate terms to the initial idea. The analysis covered the following parameters: accumulated annual production, relevance and productivity of journals and authors, and most globally cited documents. After summarizing the data, it could be proved that recycled aggregates in concrete are a growing trend, with approximately $50 \%$ of the production concentrated in the last two years. The dispersion of publications among several authors and journals is also noteworthy, so that there is a small nucleus that concentrates actions on the subject. Thus, these journals should be the target of research for future publications.

Keywords: Recycled aggregates. Concrete. Bibliometric review.

\section{Introdução}

Atualmente, um dos grandes problemas enfrentados pelo setor da construção civil ainda é o alto de índice de geração de resíduos sólidos. Por outro lado, alguns estudos demonstram a viabilidade de utilização de resíduos de construção e demolição para a produção de concretos.

Segundo Nagalli (2014), as pesquisas ainda ocorrem de maneira dispersa. A investigação sobre o aproveitamento de resíduos na construção civil também não possui sistematização e necessita de maior profundidade e padronização (YUAN; SHEN, 2011 apud NAGALLI, 2014). Desse modo, o presente trabalho tem como objetivo investigar o panorama das produções científicas, em todo o mundo, sobre a incorporação de resíduos para utilização no concreto.

\section{Fundamentação teórica}

Em consonância com Ball (2017), a bibliometria surgiu, originalmente, como um campo da biblioteconomia, com o propósito de auxiliar os bibliotecários em suas tarefas cotidianas, tais como a seleção da literatura e otimização do gerenciamento. A bibliometria, como é conhecida atualmente, remonta à primeira metade do século XX, quando questões sobre o tipo e frequência de publicações científicas começaram a adquirir maior relevância. Assim, "a ideia básica da bibliometria é quantificar a produção acadêmica de pessoas e instituições. Em uma segunda etapa, conclusões qualitativas são tomadas a partir de figuras e tabelas" (BALL, 2017, p. 15).

Em síntese, a bibliometria pode ser descrita em termos de três leis principais: a Lei de Bradford, que trata da produtividade dos periódicos; a Lei de Lotka, a qual avalia a produção científica dos autores; e a Lei de Zipf, que aborda a frequência de palavras (GUEDES, 2012). Devido à especificidade desta última, com frequência, apenas as duas primeiras leis são consideradas.

A Lei de Lotka remete a 1926, quando o matemático Alfred James Lotka descobriu que o número de artigos publicados por um autor decai de acordo com a lei do inverso do quadrado. Do ponto de vista prático, pode-se dizer que a maioria dos cientistas publica apenas uma vez, enquanto uma pequena parte contribui significativamente (BOOKSTEIN, 1980).

Já a Lei de Bradford foi estabelecida no ano de 1934, inicialmente como uma preocupação do matemático Samuel Clement Bradford em analisar se os artigos estavam 
concentrados em um número relativamente pequeno de periódicos ou se estavam distribuídos em um amplo intervalo de periódicos. Bradford compreendeu que quando um conjunto de periódicos centralizam os artigos sobre um determinado tema, os outros periódicos dividem-se em zonas decrescentes com o mesmo número de periódicos. Por conseguinte, mais e mais periódicos são necessários para produzir o mesmo número de artigos do que o núcleo (BOOKSTEIN, 1980).

Como indicador da qualidade das produções científicas, há ainda o índice-h. Segundo Hirsch (2005) apud Rousseau, Egghe e Guns (2018), se um cientista possui um índice-h igual à $h$, então todas as primeiras $h$ publicações (organizadas cronologicamente) receberam um número $h$ de citações. Como o índice-h depende diretamente do tempo, pode ser, também, um modo de estudar indiretamente a carreira do pesquisador (ROUSSEAU; EGGHE; GUNS, 2018).

\section{Metodologia}

De acordo com Gil (2008), trata-se de uma pesquisa aplicada, uma vez que visa à aquisição de conhecimentos para aplicação prática no âmbito da construção civil. Do ponto de vista dos seus propósitos, trata-se de uma pesquisa descritiva, dado que se dedica exclusivamente a estudar a produção científica internacional sobre o uso de agregados reciclados no concreto, assim como procurar possíveis relações entre os diferentes temas. Por fim, pode-se dizer que consiste em uma revisão bibliográfica, com ênfase na bibliometria.

\section{Programa experimental}

A busca de publicações restringiu-se a duas bases de dados: Scopus e Web of
Science. Ambas são bases utilizadas internacionalmente, além de facilitar a exportação de metadados para análises mais aprofundadas. Segundo o com o objetivo do trabalho, definiram-se os seguintes termos de busca: ((recycled aggregate $\mathrm{OR}$ agregado reciclado) AND (substitution OR substituição) AND (coarse aggregate OR agregado graúdo) AND (concrete OR concreto) AND (strength OR resistência)). Os idiomas utilizados foram o inglês e o português, a fim de filtrar o maior número de publicações entre o período de 2013 e 2020.

Após a aquisição dos metadados em formato adequado para a leitura, foi utilizada a biblioteca bibliometrix (ARIA; CUCCURULO, 2017), originalmente desenvolvida para a linguagem de programação R. Nesse caso, os resultados podem ser visualizados facilmente em uma página do navegador, sem maiores esforços para a escrita de códigos robustos. Posteriormente, os dados foram exportados em formato CSV (Comma Separated Values) para a realização da etapa de sumarização e apresentação dos resultados em forma de gráficos e/ou tabelas.

Analisaram-se os seguintes itens: produção anual acumulada, relevância e produtividade de periódicos e autores, além dos documentos mais citados globalmente nas duas bases de dados consultadas. Cabe ressaltar que a análise se dedica estritamente ao tema da utilização de agregados reciclados para a produção de concreto, assim como a sua influência na resistência mecânica do material e, portanto, índices gerais são apresentados em separado.

\section{Resultados e discussões}

A presente seção descreve, objetivamente, os resultados obtidos da pesquisa bibliométrica sobre a incorporação de resíduos para a produção de concretos, conforme 
os objetivos traçados inicialmente. Desse modo, apresentam-se os dados referentes às buscas nas bases de dados Scopus e Web of Science, com ênfase nos periódicos, autores e documentos publicados.

A partir dos termos ((recycled aggregate OR agregado reciclado) AND (substitution OR substituição) AND (coarse aggregate OR agregado graúdo) AND (concrete OR concreto) AND (strength OR resistência)), a busca na base de dados Scopus resultou em 61 publicações, distribuídas em 33 periódicos e entre 203 autores. Já a busca na Web of Science resultou em 39 documentos, divididos entre 39 periódicos diferentes e entre 126 autores.

\section{Produção anual científica acumulada}

Em um primeiro momento, os dados foram separados por ano de publicação, de modo a pontuar a evolução das pesquisas ao longo do tempo, de 2014 a 2020. Para efeito de comparação, os gráficos das duas bases são apresentados conjuntamente na Figura 1.

Figura 1 - Número acumulado de publicações para as bases de dados

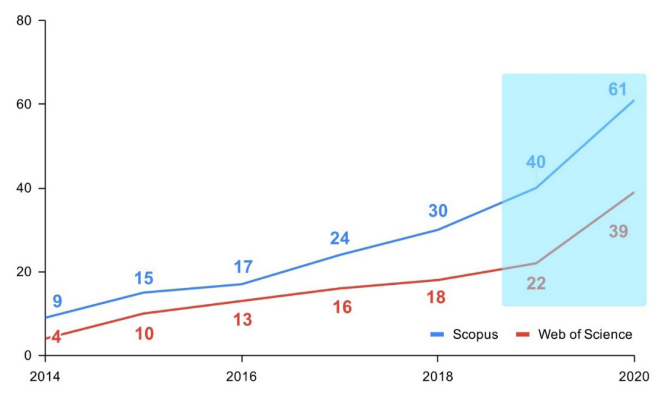

Há uma tendência de crescimento linear (média de aproximadamente 9 publicações/ ano e 6 publicações/ano) entre os anos de 2014 e 2019, com um leve predomínio do Scopus sobre a Web of Science. Contudo, entre os anos de 2019 e 2020, há um salto considerável no número de publicações, o que corrobora a importância da temática para o desenvolvimento do ambiente construído.

\section{Relevância e produtividade dos periódicos}

Os periódicos foram avaliados quanto ao número de citações, índice-h e Lei de Bradford, fatores que, em conjunto, propiciam o mapeamento adequado das fontes das bases de dados.

Os 61 artigos indexados no Scopus distribuem-se em 33 periódicos, porém de modo bastante heterogêneo. O periódico Construction and Building Materials responde por 16 publicações $(26,23 \%)$, enquanto os demais contam com até 3 publicações cada. Dentre os periódicos, a maior parte possui baixo índice-h, seja devido ao baixo número de publicações e/ou citações sobre o tema. Por outro lado, o índice geral (entre parênteses) permite concluir que os artigos vêm sendo publicados em periódicos qualificados, como mostra a Tabela I.

Ao considerar o índice-h para análise, destacam-se os seguintes periódicos: Construction and Building Materials, Materials, Engineering Structures, as quais juntas reúnem $81,96 \%$ de todas as citações em apenas $36,07 \%$ das publicações. Respectivamente, possuem índice-h de 10, 3 e 2 . Um mesmo estudo foi feito acerca dos periódicos indexados na Web of Science, cujos resultados são elencados na Tabela II.

Destaca-se novamente o periódico Construction and Building Materials, com um alto número de citações entre as publicações indexadas. $\mathrm{O}$ fato de o índice-h ser menor que o número de publicações indica, apenas, que se tratam de artigos recentes, com menor visibilidade. Em comparação com a Tabela I, o índice-h geral mostra que há maior dispersão da produção em torno de diferentes fontes. Tal comportamento pode ser mais 
Tabela I- Índice-h dos periódicos indexados na base de dados Scopus

\begin{tabular}{lccc}
\hline \multicolumn{1}{c}{ PERIÓDICO } & ÍNDICE-H & CITAÇÕES & PUBLICAÇÕES \\
\hline Construction and Building & $10(147)$ & 576 & 16 \\
Materials & $3(98)$ & 33 & 3 \\
Materials & $2(128)$ & 136 & 3 \\
Engineering Structures & $1(132)$ & 84 & 1 \\
Composites Part B: Engineering & & & \\
\hline
\end{tabular}

Tabela II - Índice-h dos periódicos indexados na base de dados Web of Science

\begin{tabular}{lccc}
\hline PERIÓDICO & ÍNDICE-H & CITAÇÕES & PUBLICAÇÕES \\
\hline Construction and Building Mate- & $7(147)$ & 222 & 11 \\
rials & $2(128)$ & 123 & 3 \\
Engineering Structures & $2(98)$ & 29 & 3 \\
Materials & $2(37)$ & 4 & 2 \\
Journal of Material Cycles and & $1(23)$ & 48 & 1 \\
$\begin{array}{l}\text { Waste Management } \\
\text { International Journal of Concrete }\end{array}$ & & \\
Structures and Materials & &
\end{tabular}

bem visualizado mediante a aplicação da Lei de Bradford para periódicos, como mostra a Figura 2.

Figura 2 - Lei de Bradford aplicada à base de dados Scopus.

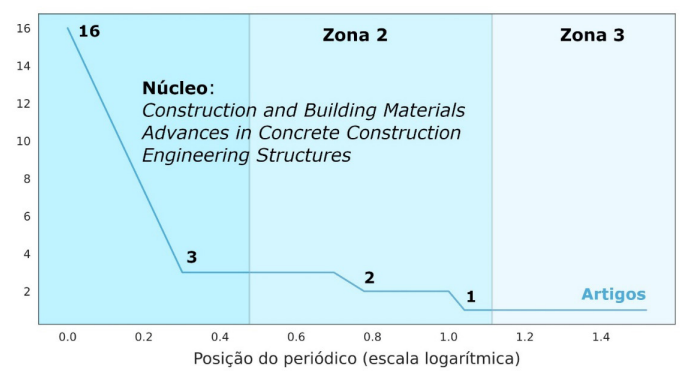

Observa-se um decréscimo acentuado da produção do núcleo em comparação às demais zonas. O núcleo concentra o maior número de publicações, com uma rápida dispersão entre os demais periódicos. A Lei de Bradford também é válida para os dados da Web of Science (Figura 3).
Figura 3 - Lei de Bradford aplicada à base de dados Web of Science

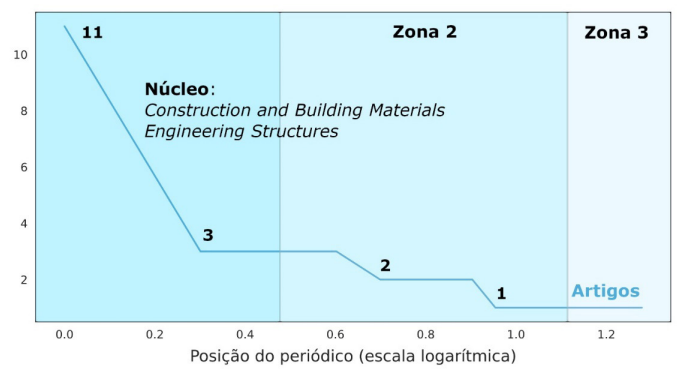

Pode-se observar que apenas dois periódicos concentram cerca de um terço das publicações. Quanto à zona 3, cabe também salientar que cada periódico possui apenas uma publicação indexada, o que indica o grau de dispersão da produção científica. Em comparação com os dados do Scopus, os resultados são bem similares, sendo os periódicos Construction and Building Materials, Advances in Concrete Construction e Engineering Structures os grandes núcleos 
de publicação sobre a incorporação de agregados reciclados em concretos.

\section{Relevância e produtividade dos autores}

A relevância e a produtividade dos autores foram avaliadas por meio do índice-h e da Lei de Lotka, que guarda uma relação bastante similar com a Lei de Bradford para os periódicos, com foco na mensuração do decréscimo da produção científica. Os dois métodos foram aplicados simultaneamente aos resultados retornados pelas duas bases de dados.
Assim como ocorre com os periódicos, são poucos os autores que concentram a produção em um determinado tema. A Tabela III apresenta o índice-h como indicador principal da relevância dos autores indexados na base de dados Scopus. Indicadores gerais de cada pesquisador são apresentados entre parênteses.

Apenas um pequeno grupo de autores possui índice-h sobre o tema mais elevado, o equivalente a menos de $5 \%$ em termos percentuais. A maior parte $(63,05 \%)$ possui apenas uma publicação sobre o tema, a qual é bastante citada e, por isso, retorna um índice-h igual a 1. A análise do índice-h também

Tabela III - Índice-h dos autores indexados na base de dados Scopus

\begin{tabular}{lccc}
\hline AUTOR & ÍNDICE-H & CITAÇÕES & PUBLICAÇÕES \\
\hline Jie Chen & $2(7)$ & $134(313)$ & $2(19)$ \\
Yuyin Wang & $2(22)$ & $135(1443)$ & $3(22)$ \\
Jianhe Xie & $2(18)$ & $88(859)$ & $2(56)$ \\
Miren Etxeberria & $2(19)$ & $85(2507)$ & $2(39)$ \\
George Wardeh & $2(13)$ & $39(450)$ & $2(32)$ \\
Francisco Baeza-Brotons & $2(8)$ & $30(251)$ & $2(17)$ \\
Nilson Santana de Amorim & $2(3)$ & $24(39)$ & $2(7)$ \\
Júnior & $2(12)$ & $24(559)$ & $2(45)$ \\
Daniel Véras Ribeiro & $2(2)$ & $24(30)$ & $2(2)$ \\
Guilherme Augusto de Oliveira & $2(11)$ & $11(422)$ & $2(77)$ \\
e Silva & & & \\
Zhiheng Deng & &
\end{tabular}

Tabela IV - Índice-h dos autores indexados na base de dados Web of Science

\begin{tabular}{lccc}
\hline AUTOR & ÍNDICE-H & CITAÇÕES & PUBLICAÇÕES \\
\hline Yuyin Wang & $3(18)$ & $158(854)$ & $3(58)$ \\
Jianhe Xie & $2(17)$ & $122(926)$ & $2(65)$ \\
Zhiheng Deng & $2(8)$ & $11(246)$ & $3(46)$ \\
Bing Liu & $2(4)$ & $4(35)$ & $2(7)$ \\
Ping Xiang & $2(14)$ & $4(485)$ & $2(53)$ \\
Bailong Ye & $2(3)$ & $4(23)$ & $2(11)$ \\
Yue Geng & $2(19)$ & $77(1030)$ & $1(76)$ \\
\hline
\end{tabular}


permite observar alguns padrões de grupos de pesquisa, como o grupo composto por Nilson Santana de Amorim Junior, Daniel Véras Ribeiro e Guilherme Augusto de Oliveira e Silva. Por outro lado, pode-se fazer uma correlação positiva entre o índice-h geral e específica sobre o assunto, dado o número de citações e publicações. Na sequência, são mostrados os índice-h dos autores indexados na Web of Science (Tabela IV).

Os dados da Web of Science também permitem identificar um grupo de pesquisa, o qual é formado por Bing Liu, Ping Xiang e Bailong Ye. Em sua maioria, esse comportamento é típico do desdobramento de um trabalho em várias publicações ou mesmo da continuidade das pesquisas. Quando comparadas as duas bases de dados, o Scopus possui autores com maior relevância, tanto no que se refere às citações quanto ao número de artigos publicados.

Quanto à produtividade dos autores, foi avaliada de acordo com a Lei de Lotka, segundo a qual o número de publicações dos autores decresce de acordo com a lei do inverso do quadrado. Os resultados para as bases de dados foram sumarizados e podem ser visualizados na Tabela $\mathrm{V}$.

Nesse caso, as duas bases de dados denotam um comportamento similar, bastante próximo à lei do inverso do quadrado. Assim, é mais notória a colaboração dos autores em trabalhos indexados na Web of Science, o que não implica necessariamente em maior qualidade. Com base na afiliação de cada autor, é possível também avaliar a produção científica em âmbito mundial, conforme mostra a Figura 4.

Tabela V - Lei de Lotka aplicada às bases de dados

\begin{tabular}{ccc}
\hline PUBLICAÇÕES & \multicolumn{2}{c}{ BASE DE DADOS } \\
\hline & Scopus & Web of Science \\
\hline 2 & $88,18 \%$ & $84,13 \%$ \\
3 & $11,33 \%$ & $11,90 \%$ \\
\hline
\end{tabular}

Figura 4 - Produção científica por país de origem

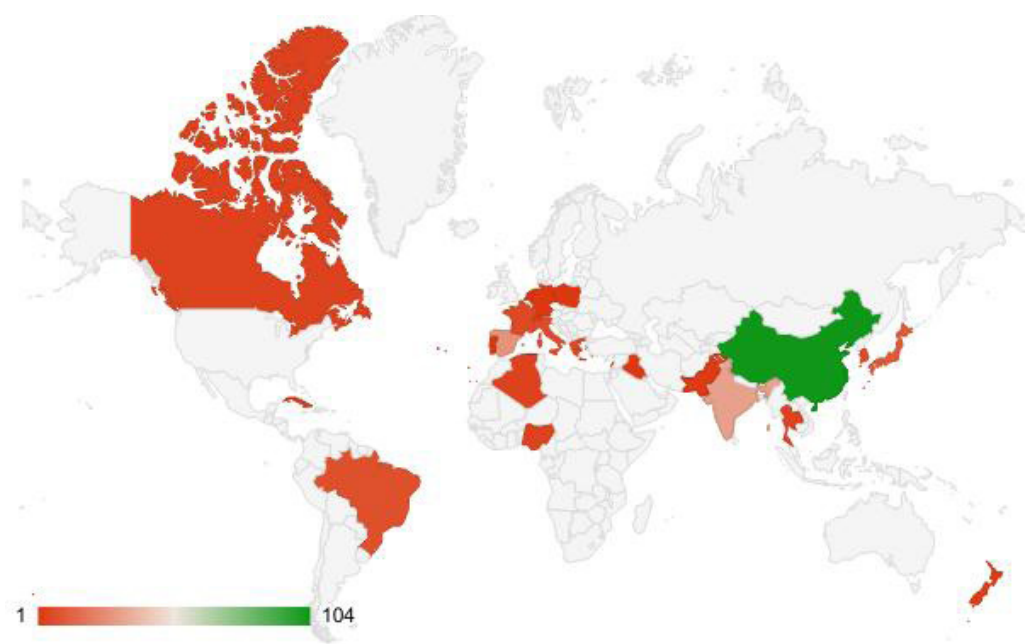


Verifica-se que a produção científica se concentra especialmente no continente asiático, em especial na China $(43,33 \%$ dos autores) e Índia $(13,33 \%)$, países que se caracterizam pela enorme população que abrigam e, portanto, com os problemas decorrentes dessa situação. Destaca-se também a Espanha, com produção similar à Índia. Quando combinados, os três países respondem por $68,33 \%$ de todas as produções e despontam como grandes celeiros no que se refere à pesquisa sobre concretos com a incorporação de agregados reciclados. $\mathrm{O}$ Brasil aparece somente na sétima posição, ainda com pouco destaque.

\section{Documentos mais citados globalmente}

Em relação aos documentos em si, a análise de citações ainda é o fator mais importante quando se verifica a influência de um trabalho sobre um determinado tema. Desse modo, a Tabela VI apresenta o número de citações para a base de dados Scopus.

Os documentos mais citados guardam uma estreita relação com a relevância e a produtividade dos autores, com exceção da publicação única de Andreu, G. e Miren, E.

Tabela VI - Documentos mais citados na base de dados Scopus

\begin{tabular}{|c|c|c|c|}
\hline AUTORES & TÍTULO & ANO & CITAÇÕES \\
\hline Andreu G., Miren E. & $\begin{array}{l}\text { Experimental analysis of properties of high } \\
\text { performance recycled aggregate concrete }\end{array}$ & 2014 & 140 \\
\hline $\begin{array}{l}\text { Wang Y., Chen J., Geng } \\
\text { Y. }\end{array}$ & $\begin{array}{c}\text { Testing and analysis of axially loaded normal-strength } \\
\text { recycled aggregate concrete filled steel tubular stub } \\
\text { columns }\end{array}$ & 2015 & 86 \\
\hline $\begin{array}{l}\text { Xie J.-H., Guo Y.-C., } \\
\text { Liu L.-S., Xie Z.-H. }\end{array}$ & $\begin{array}{c}\text { Compressive and flexural behaviours of a new steel- } \\
\text { fibre-reinforced recycled aggregate concrete with } \\
\text { crumb rubber }\end{array}$ & 2015 & 84 \\
\hline
\end{tabular}

Tabela VII - Documentos mais citados na base de dados Web of Science

\begin{tabular}{|c|c|c|c|}
\hline AUTORES & TÍTULO & ANO & CITAÇÕES \\
\hline $\begin{array}{l}\text { Wang Y., Chen J., } \\
\text { Geng Y. }\end{array}$ & $\begin{array}{l}\text { Testing and analysis of axially loaded normal-strength } \\
\text { recycled aggregate concrete filled steel tubular stub } \\
\text { columns }\end{array}$ & 2015 & 77 \\
\hline $\begin{array}{l}\text { Xie J.-H., Guo Y.-C., } \\
\text { Liu L.-S., Xie Z.-H. }\end{array}$ & $\begin{array}{l}\text { Compressive and flexural behaviours of a new steel- } \\
\text { fibre-reinforced recycled aggregate concrete with } \\
\text { crumb rubber }\end{array}$ & 2015 & 71 \\
\hline $\begin{array}{l}\text { Wardeh, G., Ghorbel, } \\
\text { E., Gomart, H }\end{array}$ & $\begin{array}{l}\text { Mix design and properties of recycled aggregate } \\
\text { concretes: } \\
\text { applicability of Eurocode } 2\end{array}$ & 2015 & 48 \\
\hline
\end{tabular}


Em geral, são artigos que datam de 2014 e 2015 e, portanto, com maior período de acesso para publicações. Quanto aos dados da Web of Science, há algumas diferenças, como mostrado na Tabela VII.

Dos 3 artigos mais citados na Web of Science, as duas primeiras posições são ocupadas por artigos também no Scopus e com um número de citações bastante semelhantes. Ou seja, dado o período de busca considerado, podem ser considerados artigos seminais para pesquisas com a mesma temática. Nesse caso, a indexação em bases de dados diferentes torna-se um agente facilitador tanto para o autor, que consegue obter maior alcance, quanto para o pesquisador, que consegue obter publicações de seu interesse com maior facilidade.

\section{Conclusão}

Por meio da revisão bibliométrica, pôde-se comprovar que o uso de agregados reciclados para a produção de concretos tem se mostrado um importante campo do conhecimento, sendo metade dos artigos publicados somente nos últimos dois anos. Logo, do ponto de vista prático, ainda há lacunas a serem preenchidas por pesquisas experimentais.

Quanto aos periódicos, há uma forte concentração em torno de um núcleo de periódicos, sendo estes mais indicados para futuras publicações. Por outro lado, países que publicam menos sobre o assunto, situação na qual se encaixa o Brasil, surgem como possíveis nichos de pesquisa. A análise de publicações por autores revelou serem poucos os autores que dedicam maiores atenções ao assunto, com grande dispersão dos dados. Já os documentos apresentaram pouca variação entre as bases, que, para este caso, não consistiram em fatores intervenientes para a revisão bibliométrica como um todo.

\section{REFERÊNCIAS}

ANDREU, G.; MIREN, E. Experimental analysis of properties of high performance recycled aggregate concrete. Construction and Building Materials, v. 52, p. 227-235

ARIA, M.; CUCCURULLO, C. bibliometrix: An R-tool for comprehensive science mapping analysis. Journal of Informetrics, v. 11, n. 4, p. 959-975, 2017.

BALL, R. An Introduction to Bibliometrics: New Development and Trends. Cambridge, Chandos: 2017.

BOOKSTEIN, A. Explanations of the Bibliometric Laws. Collection Management, v. 3, n. 2-3, p. 151-162, 1980.

GUEDES, V. L. DA S. A. BIBLIOMETRIA E A GESTÃO DA INFORMAÇÃO E DO

CONHECIMENTO CIENTÍFICO E TECNOLÓGICO: uma revisão da literatura. Pontodeacesso, v. 6, n. 2, p. 74-109, 2012.

NAGALLI, A. Gerenciamento de resíduos sólidos na construção civil. São Paulo: Oficina de Textos, 2014.

ROUSSEAU, R.; EGGHE, L.; GUNS, R. Becoming Metric-Wise: A Bibliometric Guide for Researchers. Cambridge, Chandos: 2018. 
XIE, J. H.; GUO, Y. C.; LIU, L. S.; XIE, Z. H. Compressive and flexural behaviours of a new steel-fibre-reinforced recycled aggregate concrete with crumb rubber. Construction and Building Materials, v. 79, p. 263-272, 2015.

WANG, Y.; CHEN, J.; GENG, Y. Testing and analysis of axially loaded normal-strength recycled aggregate concrete filled steel tubular stub columns. Engineering Structures, v. 86, p. 192-212, 2015 .

WARDEH, G.; GHORBEL, E.; GOMART, H. Mix Design and Properties of Recycled Aggregate Concretes: Applicability of Eurocode 2. International Journal of Concrete Structures and Materials, v. 9, n. 1, p. 1-20, 2015. 